\title{
Finding Malicious One in Group Data Sharing
}

\author{
G. Navya \\ Department of Computer Science \\ and Engineering \\ Sreenidhi Institute of Science \\ Technology, Ghatkesar
}

\author{
G. Ravi \\ Department of Computer Science \\ and Engineering \\ Sreenidhi Institute of Science \\ Technology, Ghatkesar
}

\author{
Prasanta Kumar Sahoo, PhD \\ Department of Computer Science \\ and Engineering \\ Sreenidhi Institute of Science \\ Technology, Ghatkesar
}

\begin{abstract}
Cloud computing is one of the fastest developing in the internet, it provides user demanding resources and services with low cost. Users can easily use cloud resource to do their works without any interruptions like doing personal works, social networks, video editing and business works like many more. Group data sharing grabbed people attention to share their works and thoughts in cloud computing. In this concept multiple users can access same data or documents to do works for developed of projects. With this concept users get lots of benefits because they can do work any where as they like with their resources because they don't need to buy new hardware or software to their works. Whenever group of people working on some important work then owner can't find malicious user in it. Its big challenge in cloud computing. Group members can share information anonymously without revealing their identity but this became big challenge in computing because when user used fake id's then it will be threat to document because they might misuse it. Dynamic changes of the group member, exploiting the key understanding and efficient get to control, the computational multifaceted nature and correspondence intricacy for refreshing the normal meeting key and the encryption information are generally low and its time taking process to share data in groups and consume high memory. Proposing user defined encryption for data sharing to secure communication. It can share the data to users in secure way and efficiently and tracing malicious user in group data sharing. It requires low amount to memory and low time to process encryption.
\end{abstract}

\section{Keywords}

Cloud Computing, Group Data Sharing,AES(Advanced Encryption Standard ) SBIBD(Symmetric Balanced Incomplete Block Design)

\section{INTRODUCTION}

Anonymity means person acts as unknown or who doesn't has name or identity is known as anonymous. Big challenge is their identity is not revealing and they were untraceable and unpredictable. Namelessness may lessen the responsibility one sees to have for their activities, and expels the effect these activities may somehow or another have on their notoriety. This can have emotional impacts, both valuable and hurtful to different gatherings included. In this way, it might be utilized for mental strategies including any particular gathering to indicate or support or ruin any kind of movement or conviction. Relative namelessness is regularly appreciated in huge groups.

Various individuals have distinctive mental and philosophical responses to this improvement, particularly as an advanced marvel. This obscurity is a significant factor in swarm brain research, and conduct in circumstances, for example, a mob. This apparent namelessness can be undermined by advances, for example, photography. Oblivious obedience conduct and similarity are likewise viewed as a set up impact of web namelessness. Most editorial on the Internet is basically done namelessly, utilizing unidentifiable nom de plumes. While these usernames can take on their very own character, they are much of the time isolated and mysterious from the genuine creator. As per the University of Stockholm this is making more opportunity of articulation, and less responsibility.

Anonymizing administrations, for example, I2P and Tor address the issue of IP following. To put it plainly, they work by encoding bundles inside numerous layers of encryption. The bundle finishes a foreordained course the anonymizing system. Every switch considers the to be past switch as the source and the quick next switch as the goal. Accordingly, no switch ever knows both the genuine birthplace and goal of the parcel. This makes these administrations more secure than concentrated anonymizing administrations. Assembled information are information shaped by collecting singular perceptions of a variable into gatherings, with the goal that a recurrence conveyance of these gatherings fills in as a helpful methods for outlining or dissecting the information. Data sharing is the demonstration of making data used for keen research available to various agents. Many subsidizing offices, establishments, and distribution scenes have approaches in regards to information sharing since straightforwardness and receptiveness are considered by numerous individuals to be a piece of the logical strategy. Information and techniques might be mentioned from a creator years after production. So as to support information sharing and forestall the misfortune or defilement of information, various financing offices and diaries set up approaches on information chronicling. Access to openly documented information is an ongoing improvement throughout the entire existence of science made conceivable by mechanical advances in correspondences and data innovation. To exploit present day quick correspondence may require consensual concurrence on the criteria fundamental shared acknowledgment of separate commitments.

Regardless of strategies on information sharing and documenting, information retaining still occurs. Creators may neglect to document information or they just file a bit of the information. Inability to file information alone isn't information retaining. At the point when a specialist demands extra data, a creator in some cases will not give it. At the point when creators retain information like this, they risk losing the trust of the science network. Some exploration associations feel especially unequivocally about information sharing. Stanford University's WaveLab has a way of thinking about reproducible research and uncovering all calculations and source code important to imitate the examination. In a paper titled "WaveLab and Reproducible Research," the creators portray a portion of the issues they experienced in attempting to replicate their own examination after a timeframe. As a rule, it was so troublesome they surrendered 
the exertion. Supporters of open and hybrid fogs note that disseminated processing licenses associations to avoid or restrict ahead of time IT establishment costs.

Backers similarly ensure that circulated figuring grants endeavors to get their applications completely operational speedier, with improved reasonableness and less help, and that it enables IT gatherings to even more rapidly adjust resources for fulfill fluctuating and inconsistent need, giving the burst enrolling capacity: high handling power at explicit occasions of apex demand. The objective of dispersed enlisting is to permit clients to take advantage by these advances, without the essential for noteworthy information about or limit with all of them. The cloud means to diminish expenses, and enables the clients to concentrate on their center business as opposed to being ruined by IT impediments. Virtualization gives the deftness required to revive IT endeavors, and reduces cost by developing structure use. Autonomic enrolling robotizes the procedure through which the client can strategy assets on-request. By compelling client responsibility, robotization animates the framework, lessens work costs and decreases the chance of human slip-ups.

Security can enhance record of centralization of information, broadened security-centered assets, and so forth., at any rate concerns can continue about loss of authority over certain fragile information, and the nonattendance of security for put aside bits. Security is reliably in a comparative class as or superior to other standard frameworks, fairly since star affiliations can submit points of interest for explaining security gives that different clients can't stay to manage or which they come up short on the specific aptitudes to address.

\section{RELATED WORKS}

A key understanding show is used to create a commonplace social affair key for various individuals to ensure the security of their later correspondences, and this show can be applied in dispersed figuring to help secure and capable data sharing. At any rate it can make only two keys . it doesn't give any check organization, which makes it unprotected for man-in-theinside attack. Key understanding show is made by DiffieHellman. It can reinforce only two individuals. For correspondence and data participating in bundles security levels are low.

In these item improvement circumstances, various customers in a social occasion need to share the source code, and they need to find a workable pace, and run the basic source code at whatever point and spot. An attacker outside the get-together (join the disavowed pack client passed on limit server) may increase a few information on the plaintext of the information. Really, this sort of assailant needs to at rent break the security of the got gathering information encryption think up. The passed on accumulating server thinks up with the denied pack clients, and they need to give an unlawful information without being perceived. Social event mark is presented by Chaum and Heyst .It offers mystery to guarantors, where each gettogether part has a private key that engages the customer to sign messages. In any case, the resulting mark keeps the character of the guarantor puzzle. Generally speaking, there is a pariah that can lead the imprint anonymity using an excellent trapdoor. A couple of systems support forswearing where bundle investment can be injured without affecting the stamping limit of unrevoked customers. By giving fake nuances any individual can take an interest in bundles which it will offer access to aggressors. the arrangement is a short signature plot where customer repudiation just requires sending forswearing information to signature verifiers.
These capacities are classified "single direction" since they are anything but difficult to figure one way yet (evidently) hard to register the other way. They are designated "trapentryway" capacities since the opposite capacities are in actuality simple to register once certain private "trapentryway" data is known. Two clients can likewise set up private correspondence over a shaky interchanges channel without con-fitting an open record. Since no methods exist to demonstrate that an encryption plot is secure, the main test accessible is to see whether anybody can think about an approach to break it.

Information re-appropriated to the cloud are not kept safely and still experience the ill effects of an assortment of security assaults both interior and outside. From one viewpoint, vindictive system assaults, which are outside and well-known to Internet clients, compromise cloud information. Programmers may recover and take cloud clients' information or even degenerate and erase the information, obliterating its confidentiality, uprightness, and accessibility. some current conventions are excessively costly as far as correspondence as well as calculation, different conventions may experience the ill effects of low efficiency in supporting information dynamics. the file data essentially comprises of the file ID, in that creation the length of the file ID long. In addition, when the absolute number of files increments after some time, it turns out to be more difficult to make each file ID unique. the timestamp taking part in is produced by the information proprietor itself also. That is the place the issue lies. In addition, some essential difficulties in cloud examining, clump inspecting, blockless verification and lethargic update.

\section{EXISTING SYSTEM}

In the existing system encryptions are in 64 bit which leaves user documents have low security. This is big advantage to attackers and malicious users. In this malicious user uses data to their wish. Finding malicious attacker are not available in the existing system. With this loop hole attacker are using document for their own profits. Consuming lots of resources and waste of money and time to users.

\section{PROPOSED SYSTEM}

Proposing a method tracing person in group of people whoever present in data sharing. It finds IP's address and hostname of system. When ever user update data then it shows warning message to user and when user completes update then it finds nearest geo location with the help of latitude and longitude. So admin can find the location with the help of latitude and longitude in Google maps. To secure users file server uses AES256 bit encryption to give better security for user files. If any attacker wants to hack users files then first he should know the user details. But server already encrypted data files into unreadable format. If attacker wants to decrypts then its impossible because the encryption levels are in 256bit advanced level. This AES is used by military bases to secure their communications. With this people can get more security and can easily find malicious people. 


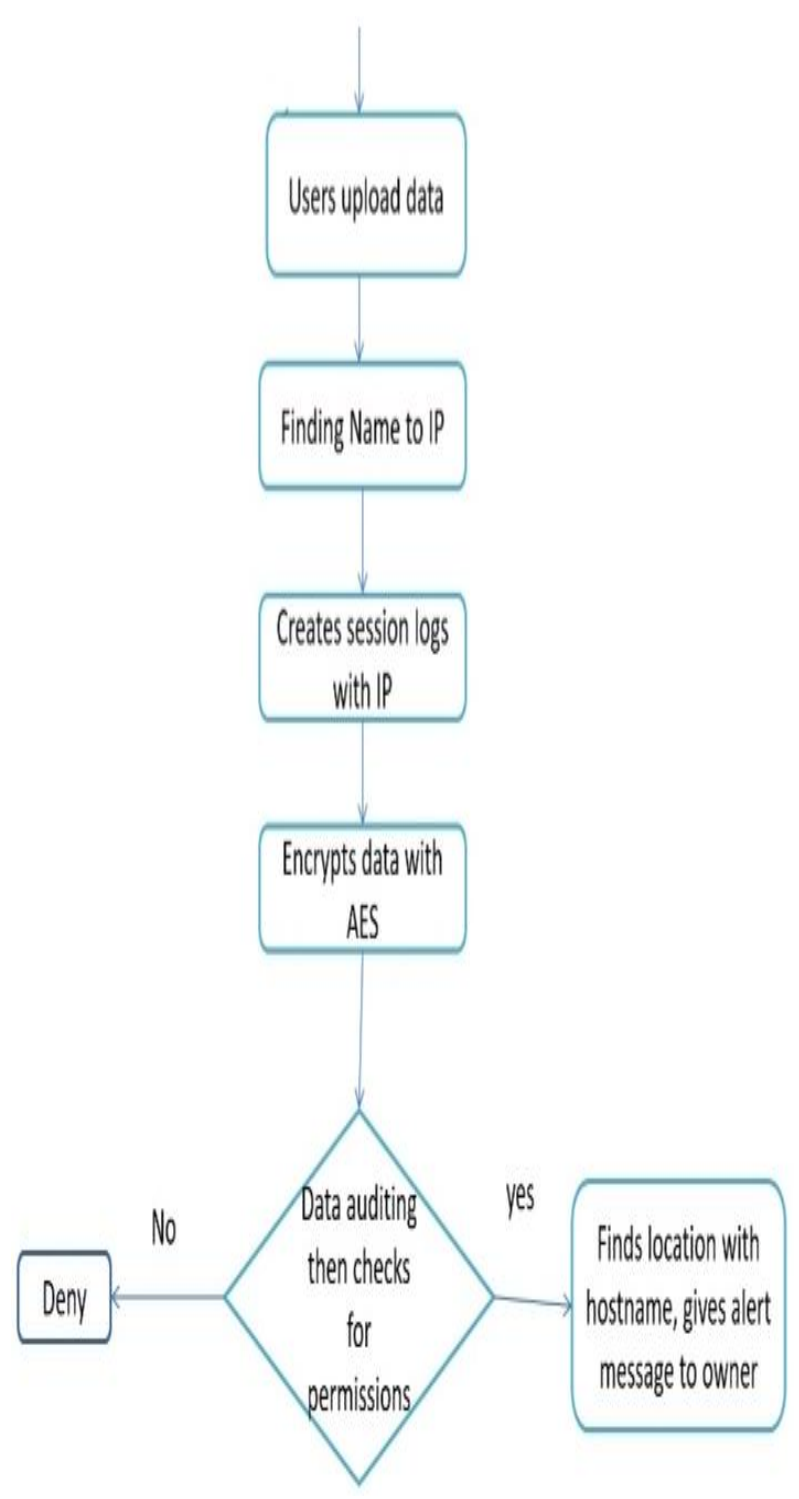

Fig-1Architecture model

\section{CONCLUSION}

Tracing malicious person developed successfully. With the proposed system users can get far better security for user documents. With this admin can get malicious persons location and IP address with hostname of system. By this way we can reduce malicious persons in sharing of group data. Proposed system will display latitude and longitude of person whoever ever update documents. It will be shown. With latitude and longitude owner can easily get location of users by using google maps. Google maps will displays the location with latitude and longitude. In the proposed system have used AES256 bit encryption to encrypt user documents in the server.

\section{REFERENCES}

[1] "Block Design-based Key Agreement for Group Data Sharing in Cloud Computing" 1545-5971 (c) 2017 IEEE. Personal use is permitted, but republication/redistribution requiresIEEEpermission.See http://www.ieee.org/publications_standards/publications/ rights/index.html for more information.

[2] "Public Integrity Auditing for Shared Dynamic Cloud Data with Group User Revocation" This article has been accepted for publication in a future issue of this journal, but has not been fully edited. Content may change prior to final publication. Citation information: DOI 10.1109/TC.2015.2389955, IEEE Transactions on Computers

[3] "A Method for Obtaining Digital Signatures and PublicKey Cryptosystems "Communications February 1978 of Volume 21 the ACM Number 2, (C) 1978 ACM 00010782178/0200-0120\$00.75 .

[4] "An Efficient Public Auditing Protocol With Novel Dynamic Structure for Cloud Data" IEEE TRANSACTIONS ON INFORMATION FORENSICS AND SECURITY, VOL. 12, NO. 10, OCTOBER 2017

[5] "A Projection-based Hotspot Analysis Method "2013 International Conference on Mechatronic Sciences, Electric Engineering and Computer (MEC) Dec 20-22, 2013, Shenyang, China.

[6] "Reduction of Malicious Behavior Patterns Based on Attribute Order" 978-1-4244-6585-9/10/\$26.00@2010 IEEE

[7] "Malicious Node Detection On Vehicular Ad-Hoc Network Using Dempster Shafer Theory For Denial Of Services Attack "2016 8th International Conference on Computational Intelligence and Communication Networks, 978-1-5090-1144-5/16 \$31.00 @ 2016 IEEE DOI 10.1109/CICN.2016.91.

[8] "Unknown Malicious Executables Detection Based on Run-time Behavior "Fifth International Conference on Fuzzy Systems and Knowledge Discovery, 978-0-76953305-6/08 \$25.00 (c) 2008 IEEE DOI 10.1109/FSKD.2008.185 .

[9] "Systematic Scanning for Malicious Source Code" 9781-4244- 1978-4/08/\$25.00 02008 IEEE

[10] "Practical Attribute-Based Encryption: Traitor Tracing, Revocation and Large Universe" The Computer Journal Advance Access published January 28, 2016, The British Computer Society 2015. All rights reserved. For Permissions,pleaseemail: journals.permissions@oup.com doi:10.1093/comjnl/bxv101. 